\title{
Caspase 4 Overexpression in Clear Cell Renal Cell Carcinoma and Its Prognostic Role
}

Lingfeng Meng

Beijing Hospital

Zijian Tian

Beijing Hospital

Xingbo Long

Beijing Hospital

Tongxiang Diao

Beijing Hospital

Maolin Hu

Beijing Hospital

Miao Wang

Beijing Hospital

Wei Zhang

Beijing Hospital

Yaoguang Zhang ( $\sim$ zhang003887@sina.com )

Beijing Hospital https://orcid.org/0000-0002-1024-9454

Jianye Wang ( $\nabla$ wangjy@bjhmoh.cn )

Beijing Hospital

Yuhui He

China-Japan Friendship Hospital

Primary research

Keywords: CASP4, clear cell renal cell carcinoma, prognosis

Posted Date: June 17th, 2020

DOl: https://doi.org/10.21203/rs.3.rs-35104/v1

License: (9) This work is licensed under a Creative Commons Attribution 4.0 International License. Read Full License 


\section{Abstract}

Background: Caspase 4 (CASP4) dysregulation is related to the occurrence, development, and outcome of many malignant tumors, but its role in clear cell renal cell carcinoma (ccRCC) is unclear. This study was conducted to investigate the expression level of CASP4 in tumor tissues and its relationship with clinical prognosis of patients with ccRCC.

Methods: First, the Oncomine and The Cancer Genome Atlas databases were used to determine CASP4 mRNA expression in ccRCC and its association with ccRCC prognosis. We then performed immunohistochemical staining and evaluation of 30 paired ccRCC and adjacent normal tissues to confirm these results. The correlation between CASP4 expression and cCRCC prognosis was evaluated using Kaplan-Meier analysis, and related genes and pathways were obtained from The Cancer Genome Atlas database by gene set enrichment analysis and gene set variation analysis. Finally, we explored the co-expression of genes with CASP4 in ccRCC.

Results: CASP4 mRNA expression in ccRCC was significantly higher than that in normal tissues $(\mathrm{p}<$ 0.001). Kaplan-Meier analysis showed that the overall survival of patients with ccRCC showing high CASP4 expression was significantly reduced $(p<0.001)$. We then used external datasets (Gene Expression Omnibus database and patients from our center) to verify the level of CASP4 expression and survival differences (all $p<0.05$ ). We also found that differential expression levels of CASP4 were correlated with pathological grade and clinical TNM stage (all $p<0.05$ ).

Conclusions: Overall, our study shows that CASP4 is highly expressed in ccRCC and is an important factor affecting prognosis. Thus, CASP4 may be a potential prognostic biomarker of CcRCC.

\section{Background}

Kidney cancer is one of the most common malignant tumors of the urinary system. More than 100,000 people worldwide die of kidney cancer annually, and more than $90 \%$ of these patients suffer from renal cell carcinoma (RCC) [1]. RCC accounts rank 7th and 9th in the incidence of malignant tumors among men and women, respectively [2]. Epidemiological investigations have shown that RCC is the second most common tumor of the genitourinary system in China after bladder tumors [3, 4]. Clear cell RCC (cCRCC) is the most common pathological type of RCC, accounting for $75-80 \%$ of cases, and its incidence in males is higher than that in females [5]. Surgery is the first-choice treatment for ccRCC in the early stage. The 5 -year survival rate after early operation is approximately $70 \%$. Around $30 \%$ of patients relapse within 5 years after surgery [6]. However, early diagnosis is difficult, and most patients require radical nephrectomy upon diagnosis. Additionally, ccRCC is not sensitive to conventional radiotherapy and chemotherapy, and its prognosis is often poor once metastasized. Therefore, studying the molecular mechanism underlying the occurrence and development of ccRCC is very important. Exploring new molecular diagnostic markers can facilitate early diagnosis and expand the understanding of disease progression with the discovery of new inhibitory targets for ccRCC. 
Caspase 4 (CASP4) is a member of the caspase (aspartate-specific cysteinyl proteinase) family of proteins. Caspases are a group of proteolytic enzymes that participate in the coordination of cellular processes including homeostasis, inflammation, and apoptosis. CASP4 disorders are known to be associated with many diseases including inflammatory bowel disease [7], Alzheimer's disease [8], Parkinson's disease [9], and various malignant tumors [10-13]. However, the relationship between CASP4 expression levels and clinical prognosis in patients with ccRCC remains unclear. The autophagy function of CASP4 and its effect on the growth and migration of tumor cells are potential targets in cancer therapy; caspases are thought to be key for developing more effective anticancer therapies. Therefore, studies of CASP4 may guide treatments aimed at improving the prognosis of ccRCC.

\section{Materials And Methods}

\section{Study aim, design, and setting}

To evaluate the expression of CASP4 in tumor tissues and determine its value in patients with ccRCC, we explored the expression, functional enrichment, and prognostic role of CASP4 in 611 patients with ccRCC from The Cancer Genome Atlas (TCGA) database, and recruited 30 patients who had previously undergone radical nephrectomy and were pathologically diagnosed with ccRCC at Beijing Hospital to verify the results. We hypothesized that CASP4 is overexpressed in patients with ccRCC, is associated with poor prognosis, and shows potential as a marker of ccRCC.

\section{Data sources}

The mRNA expression profile and corresponding clinical information of patients with ccRCC were obtained from TCGA. As of January 16, 2020, the platform contained 539 ccRCC tissues and 72 adjacent non-tumor tissues. Additionally, the GSE29609 dataset was obtained from the Gene Expression Omnibus (GEO) database (http://www.ncbi.nlm.nih.gov/geo/) and used for further bioinformatics analysis.

Thirty patients with ccRCC who underwent radical nephrectomy at Beijing Hospital from December 2012 to April 2019 were selected for the study. Immunohistochemical staining was performed on their tissue samples, and their survival information was followed up through telephone.

\section{Differential expression of CASP4 and establishment of survival and risk curve}

First, we used the Limma R package to calculate the differential expression of CASP4 in ccRCC and normal tissue samples. The results were visualized in a box diagram.

We also divided patients with cCRCC from TCGA database into low-expression and high-expression groups according to the median CASP4 expression and drew Kaplan-Meier curves to compare the survival difference between these two groups. A risk curve was generated to visualize the risk value and survival status of patients in the low-expression and high-expression groups. Finally, patients with ccRCC from 
Beijing hospital were divided into low-risk and high-risk groups according to the median immunohistochemical score (H-score), and Kaplan-Meier curves were drawn to compare their differences.

\section{Correlation between CASP4 and clinicopathological parameters and its molecular characteristics}

To explore the relationship between CASP4 expression and clinicopathological characteristics, we used the patient's age, sex, pathological grade, and clinical staging system as classification variables to explore the differences between different clinicopathological parameters and CASP4 expression.

Gene set variation analysis (GSVA) is a pathway-based analysis method that provides each sample with an overall pathway or gene set activity score. We used the GSVA R package to identify and visualize the most relevant pathway to CASP4. We also used the median CASP4 expression to divide patients into high- and low-risk groups and analyzed the groups using gene set enrichment analysis (GSEA) software v4.0 to explore the functions or pathways of CASP4 that play a role in tumorigenesis and development, and then visualized the results.

To further explore the possible molecular mechanism of CASP4, we identified genes co-expressed with CASP4 in patients with ccRCC. To obtain high-dimensional information, we used the ggplot2 and circlize $\mathrm{R}$ packages to visualize co-expressed genes and their relationships.

\section{Results}

\section{High CASP4 expression in ccRCC}

We first used the Oncomine database to determine whether the CASP4 mRNA expression level in ccRCC tissues was significantly higher than that in normal tissues (Supp. Figure 1, $p<0.001$ ). The results are consistent with those from TCGA database (Fig. 1A). To verify the true expression level of CASP4 in cCRCC, we performed immunohistochemical staining of 30 pairs of ccRCC and adjacent normal tissues from our center (Fig. 2).

\section{Correlation between CASP4 expression and survival of patients with CCRCC}

Analysis of TCGA database revealed that the survival time of patients with $\mathrm{CCRCC}$ showing high expression of CASP4 mRNA was shorter than in those with low expression of CASP4 mRNA $(p<0.001$, Fig. 1B). Simultaneously, we visualized the distribution of high and low CASP4 expression in patients with $\mathrm{ccRCC}$ and the number of patients in the high and low expression groups (Supp. Figure 2) .

In addition, samples from our center were divided into high-expression and low-expression groups according to the median $\mathrm{H}$-score, and Kaplan-Meier curves were drawn to compare survival between the two groups. The H-score was calculated as described previously [14]. Kaplan-Meier analysis showed that 
CASP4 upregulation was closely related to the overall survival (OS) of patients with ccRCC $(p<0.05$, Fig. 3A). Similar results were observed for the GSE29609 dataset ( $p<0.05$, Fig. 3B).

\section{Relationship between CASP4 expression and clinicopathological parameters}

We further explored the relationship between clinicopathological parameters and CASP4 expression. The results showed that CASP4 expression increased with an increasing pathological grade $(p<0.001$, Fig. 4A), and similar results were observed for clinical stages ( $<<0.001$, Fig. 4B). Moreover, CASP4 expression in T3-4 was higher than that in T1-2 ( $<<0.001$, Fig. 4C), higher in N1 than in N0 ( $p=0.038$, Fig. 4D), and higher in $M 1$ than in M0 ( $p<0.001$, Fig. 4E). No difference in CASP4 expression was observed between elder and younger patients $(p=0.622$; Fig. $4 F)$ or between male and female patients ( $p$ $=0.052 ;$ Fig. 4G).

\section{Functional enrichment analysis and co-expression of CASP4}

To further explore the potential function of CASP4 in CCRCC, we analyzed the RNA-Seq data of patients with cCRCC in TCGA database by GSEA and GSVA. According to the median CASP4 expression in TCGA dataset, ccRCC samples were divided into high- and low-expression groups. Notably, GSEA and GSVA analysis showed that gene sets with higher scores were significantly enriched in immune- and cell cyclerelated pathways (Fig. 5A,B).

We also explored the genes co-expressed with CASP4 in ccRCC, using an false discovery rate $<0.001$ and |log2 (fold-change)|ख0.5 as the standard, and 5 genes with the strongest positive and negative correlation were selected, respectively. The five genes showing the strongest positive correlation were CASP1 (Cor = 0.768, Fig. 6A), GMIP (Cor=0.711, Fig. 6B), APOBEC3G (Cor=0.711, Fig. 6C), MILR1 (Cor=0.71, Fig. 6D), and LPXN (Cor $=0.708$, Fig. $6 \mathrm{E})$. The five genes showing the strongest negative correlation were DYNLL2 (Cor $=-0.617$, Fig. 6F), CTDSPL (Cor $=-0.589$, Fig. 6G), NDRG2 (Cor $=-0.584$, Fig. 6H), HYAL1 (Cor = -0.583 , Fig. $6 \mathrm{l})$, and WLS (Cor $=-0.575$, Fig. $6 \mathrm{~J}$ ). Finally, we used the co-expression circle map to visualize the relationship among the above genes (Fig. 6K).

\section{Discussion}

cCRCC accounts for $2-3 \%$ of all cancers in humans, and the morbidity and mortality of ccRCC have increased significantly in the past 20 years [15]. It is estimated that more than 300,000 people are diagnosed with ccRCC each year, which is expected to increase by $22 \%$ in 2020 [16]. More than 100,000 ccRCC-related deaths are reported each year. ccRCC is a complex heterogeneous disease, and its pathogenesis is unclear. Although a large number of studies has been carried out using microarrays and RNA-Seq to identify new biomarkers and therapeutic targets for $\mathrm{ccRCC}$, there are inconsistencies between the differentially expressed genes found in different studies. Therefore, it is necessary to find a reliable and relatively simple method for prognosis. 
Analysis using the Oncomine, GEO, and TCGA databases revealed that the expression level of CASP4 mRNA in ccRCC tissues was significantly higher than that in normal tissues. To verify this result, we performed immunohistochemical staining of 30 cases of ccRCC and adjacent normal tissues at our center. The immunohistochemical results showed that the expression level of CASP4 protein in ccRCC tissues was significantly higher than that in adjacent normal tissues.

The prognostic significance of CASP4 overexpression in tumors is controversial. Wang reported that in patients with gastric cancer, higher expression of CASP4 was associated with better OS [17]. However, Terlizzi et al found that CASP4 overexpression is associated with poor prognosis in patients with nonsmall cell lung cancer [18]. The prognostic significance of CASP4 in cCRCC has not been reported. We found that the survival time of patients with ccRCC showing high CASP4 mRNA expression was shorter than that of patients with low expression levels, which was verified by GEO datasets and experimental data. We also combined the differential expression level of CASP4 with clinicopathological parameters and found that its expression was related to pathological grade and clinical TNM stage.

In contrast, to further explain the possible molecular mechanism of CASP4 in cCRCC, we used GSEA and GSVA to analyze the data obtained from public databases to identify important pathways. GSEA and GSVA analysis illustrated that CASP4 is involved in the most significant pathways including ANTIGEN_PROCESSING_AND_PRESENTATION, DNA_REPLICATION, CELL_CYCLE, and nervous system diseases, which were enriched in ccRCC samples. Genes co-expressed with CASP4 were also identified, which may help to further explore the interaction between these genes. These results indicate that CASP4 plays a key role in the occurrence and development of ccRCC.

Previous studies confirmed that CASP4 encodes a protein related to immunity and inflammation, which is involved in the coordination of cellular processes, including cell homeostasis, inflammation, and apoptosis [19]. Our study showed that CASP4 is related to the innate immune response and inflammation and is closely related to the occurrence and development of tumors. Abnormal expression of CASP4 may thus be involved in the occurrence, development, and metastasis of tumors. Studies in tumor cells will identify roles for CASP4 in addition to the traditional concepts of its role in inflammatory bodies in the immune system and reveal the interdependent or mutually restricted relationship between CASP4 and upstream regulatory factors and downstream effector molecules, as well as pyroptosis, or other pathways of cell death.

This study also had some limitations. First, this was a retrospective study, and selective bias is inevitable. Second, the sample size of the study was relatively small, and the population diversity was poor. Third, we only used immunohistochemistry (a semi-quantitative method) to detect the expression of CASP4 protein. Finally, we did not clarify the potential mechanism of CASP4 participation in ccRCC.

\section{Conclusions}

Overall, our study showed that CASP4 expression is increased in patients with ccRCC and this increased expression is significantly associated with a later clinical stage, higher pathological grade, and lower 
survival rate. Thus, CASP4 may be useful as a prognostic marker for patients with ccRCC to help clinicians make a diagnosis and treatment plans, and as a potential treatment target, which must be confirmed in further studies.

\section{List Of Abbreviations}

Caspase 4, CASP4; clear cell renal cell carcinoma, ccRCC; Gene Expression Omnibus, GEO; gene set enrichment analysis, GSEA; gene set variation analysis GSVA; immunohistochemical score, $\mathrm{H}$-score; renal cell carcinoma, RCC; The Cancer Genome Atlas, TCGA

\section{Declarations}

\section{Ethics approval and consent to participate}

All protocols were approved by the Research Ethics Committee of Beijing Hospital, and written informed consent was obtained from all participants.

\section{Consent for publication}

Not applicable.

\section{Availability of data and materials}

The datasets used and/or analyzed during the current study are available from the corresponding author on reasonable request.

\section{Competing interests}

The authors declare that they have no competing interests.

\section{Funding}

This work was supported by the National Key R\&D Program of China [Grant number 2018YFC2002202].

\section{Authors' contributions}

Dr. Meng Lingfeng and Tian Zijian drafted and revised the manuscript, Xingbo Long, Tongxiang Diao, Maolin Hu, Miao Wang, Wei Zhang, Yaoguang Zhang, Jianye Wang, and Yuhui He all contributed equally 
to study conduct, including patient recruitment, data collection, and data analysis. All authors reviewed and approved the final version of the manuscript.

\section{Acknowledgements}

Not applicable.

\section{References}

1. Ferlay J, Shin HR, Bray F, Forman D, Mathers C, Parkin DM. Estimates of worldwide burden of cancer in 2008: GLOBOCAN 2008. Int J Cancer. 2010;127:2893-917.

2. Gupta K, Miller JD, Li JZ, Russell MW, Charbonneau C. Epidemiologic and socioeconomic burden of metastatic renal cell carcinoma (mRCC): a literature review. Cancer Treat Rev. 2008;34:193-205.

3. Zhao ZW, Li YJ. Advances in epidemiology of renal cell carcinoma. Shandong Med J. 2013;53:7.

4. Jianhui MA, Ming LI, Zhang S, Yanqun NA, Chen W. Renal cancer incidence trends of cities and counties in China. Chin J Urol. 2009;30:511-4.

5. Cohen HT, McGovern FJ. Renal-cell carcinoma. N Engl J Med. 2005;353:2477-90.

6. Di Martino S, De Luca G, Grassi L, Federici G, Alfonsi R, Signore M, et al. Renal cancer: new models and approach for personalizing therapy. J Exp Clin Cancer Res. 2018;37:1-18.

7. Flood B, Oficjalska K, Laukens D, Fay J, O'Grady A, Caiazza F, et al. Altered expression of caspases-4 and -5 during inflammatory bowel disease and colorectal cancer: diagnostic and therapeutic potential. Clin Exp Immunol. 2015;181:39-50.

8. Kajiwara Y, McKenzie A, Dorr N, Gama Sosa MA, Elder G, Schmeidler J, et al. The human-specific CASP4 gene product contributes to Alzheimer-related synaptic and behavioural deficits. Hum Mol Genet. 2016;25:4315-27.

9. Arai H, Furuya T, Mizuno Y, Mochizuki H. Inflammation and infection in Parkinson's disease. Histol Histopathol. 2006;21:673-8.

10. Papoff G, Presutti D, Lalli C, Bolasco G, Santini S, Manelfi C, et al. CASP4 gene silencing in epithelial cancer cells leads to impairment of cell migration, cell-matrix adhesion and tissue invasion. Sci Rep. 2018;8:1-14.

11. Scapoli L, Girardi A, Rubini C, Martinelli M, Spinelli G, Palmieri A, et al. LOH at PDCD4, CTNNB1, and CASP4 loci contributes to stage progression of oral cancer. Int J Immunopathol Pharmacol. 2011;24:89-93.

12. Nilsson O. Profiling of ileal carcinoids. Neuroendocrinology. 2013;97:7-18.

13. Schulten HJ, Hussein D, Al-Adwani F, Karim S, Al-Maghrabi J, Al-Sharif M, et al. Microarray expression profiling identifies genes, including cytokines, and biofunctions, as diapedesis, associated with a brain metastasis from a papillary thyroid carcinoma. Am J Cancer Res. 2016;6:2140. 
14. Yeo W, Chan SL, Mo FK, Chu CM, Hui JW, Tong JH, et al. Phase I/II study of temsirolimus for patients with unresectable Hepatocellular Carcinoma (HCC)-a correlative study to explore potential biomarkers for response. BMC Cancer. 2015;15:395.

15. Ferlay J, Steliarova-Foucher E, Lortet-Tieulent J, Rosso S, Coebergh JWW, Comber H, et al. Cancer incidence and mortality patterns in Europe: estimates for 40 countries in 2012. Eur $\mathrm{J}$ Cancer. 2013;49:1374-403.

16. Ferlay J, Soerjomataram I, Dikshit R, Eser S, Mathers C, Rebelo M, et al. Cancer incidence and mortality worldwide: sources, methods and major patterns in GLOBOCAN 2012. Int J Cancer. 2015;136:E359-E386.

17. Wang Z, Ni F, Yu F, Cui Z, Zhu X, Chen J. Prognostic significance of mRNA expression of CASPs in gastric cancer. Oncol Lett. 2019;18:4535-54.

18. Terlizzi M, Colarusso C, De Rosa I, De Rosa N, Somma P, Curcio C, et al. Circulating and tumorassociated caspase-4: a novel diagnostic and prognostic biomarker for non-small cell lung cancer. Oncotarget. 2018;9:19356.

19. Krause K, Caution K, Badr A, Hamilton K, Saleh A, Patel K, et al. CASP4/caspase-11 promotes autophagosome formation in response to bacterial infection. Autophagy. 2018;14:1928-42.

\section{Figures}
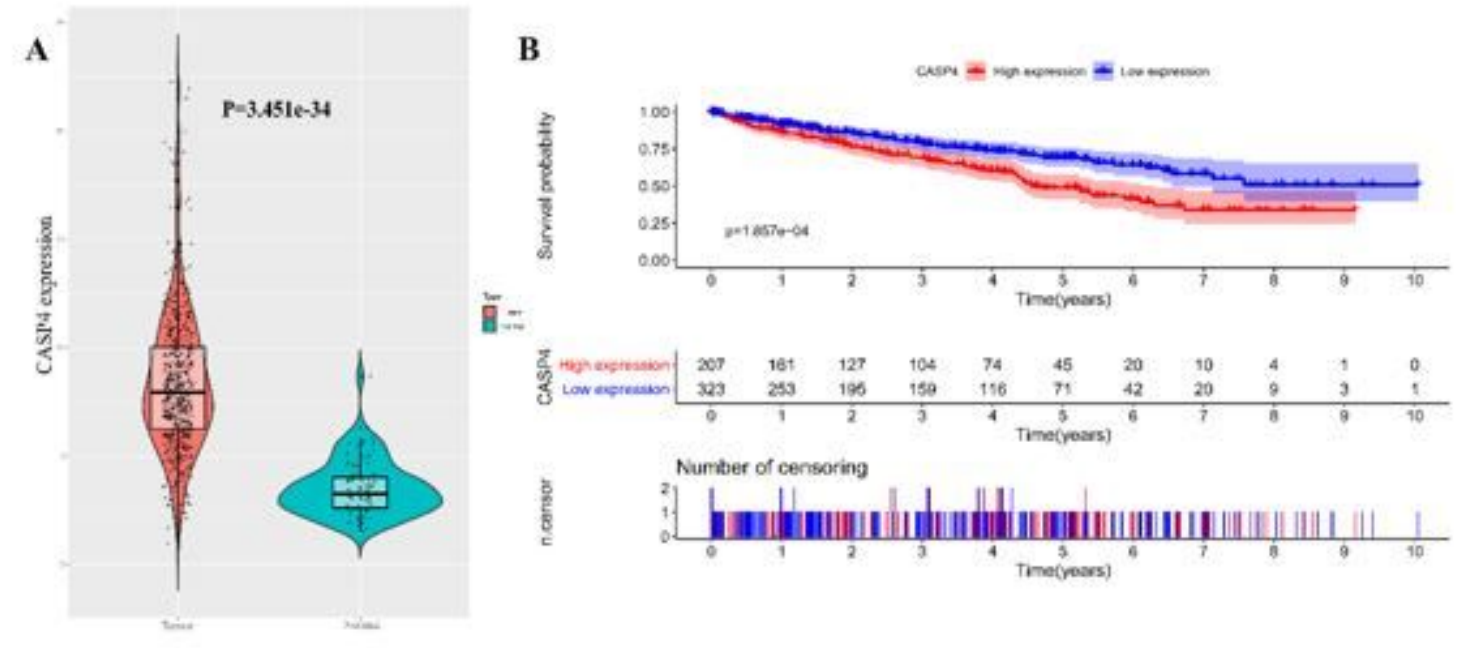

\section{Figure 1}

Difference in CASP4 expression in patients with cCRCC and its relationship with prognosis in the TCGA database. (A) Difference in CASP4 expression between ccRCC and normal tissues. (B) OS of patients with high CASP4 expression was significantly lower than that of patients with low CASP4 expression. 


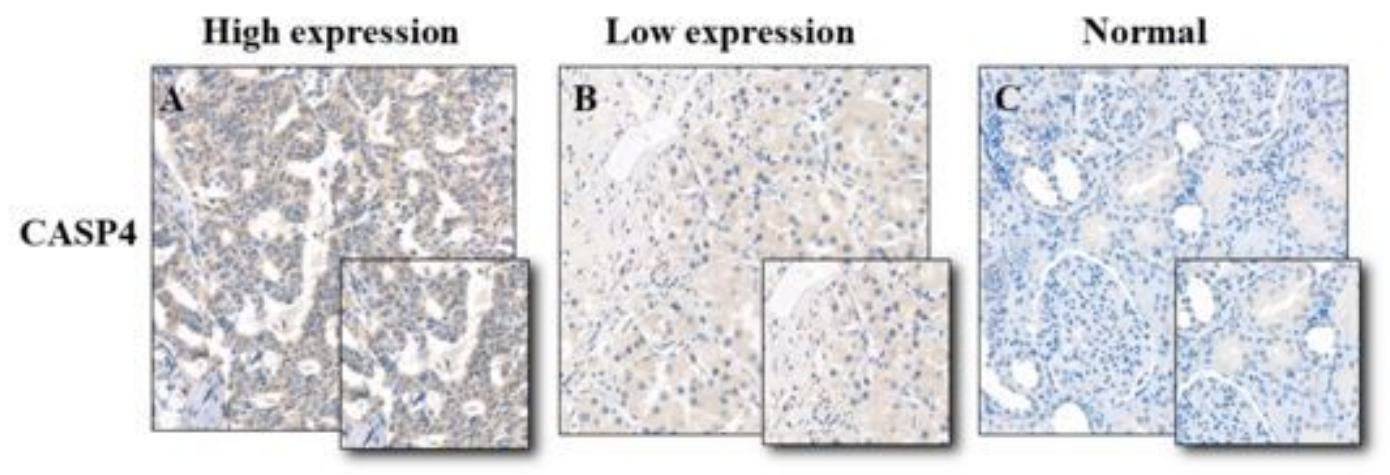

Figure 2

Immunohistochemical staining of CASP4 in ccRCC and adjacent normal tissues. (A) High CASP4 expression in ccRCC tissues; (B) Low CASP4 expression in ccRCC tissues; (C) Low CASP4 expression in adjacent normal tissues.
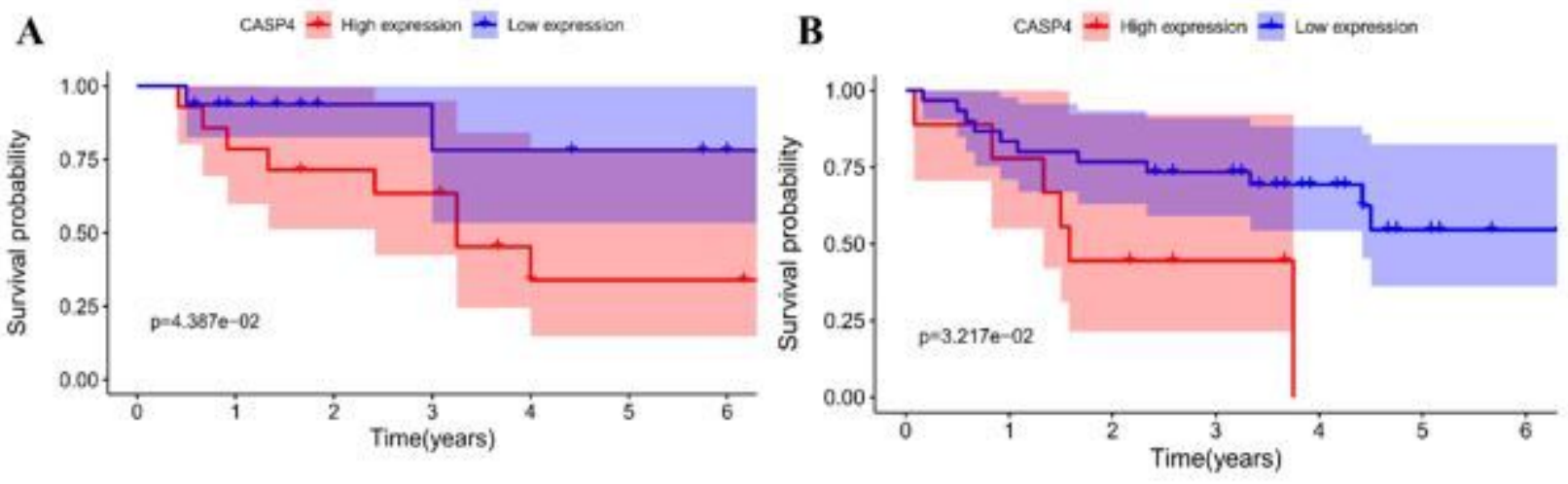

\section{Figure 3}

Survival differences of patients with different CASP4 expression in external dataset. (A) Based on the $\mathrm{H}$ Score value of CASP4, the OS of high-risk patients was significantly lower than that of low-risk patients. (B) K-M analysis of patients with high and low expression of CASP4 in the GSE29609 data set. 
A
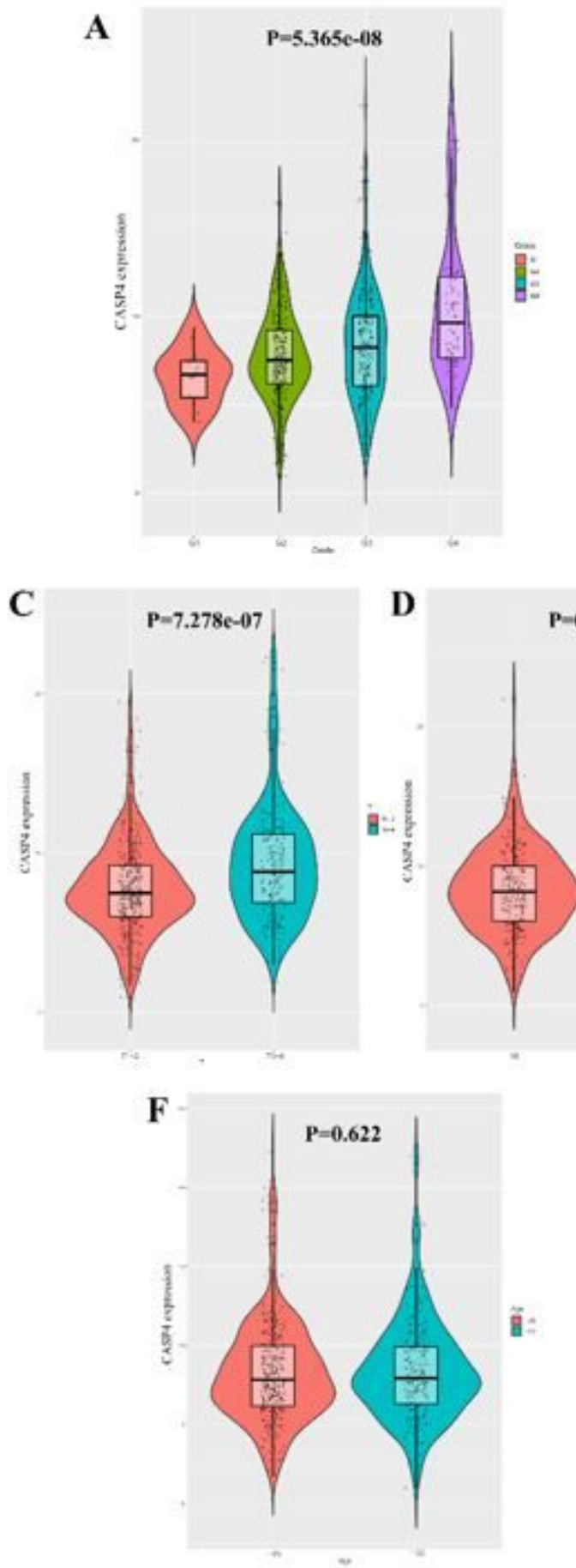

B
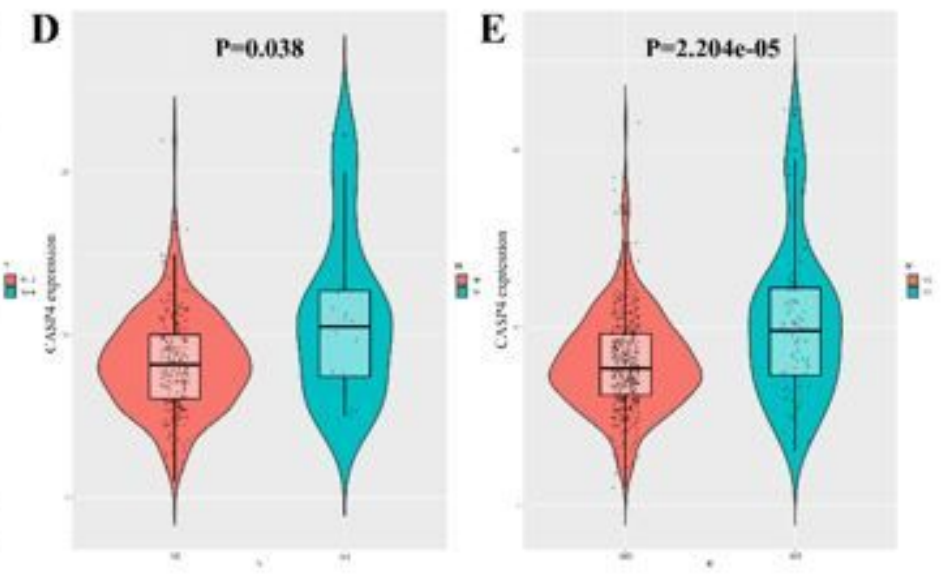

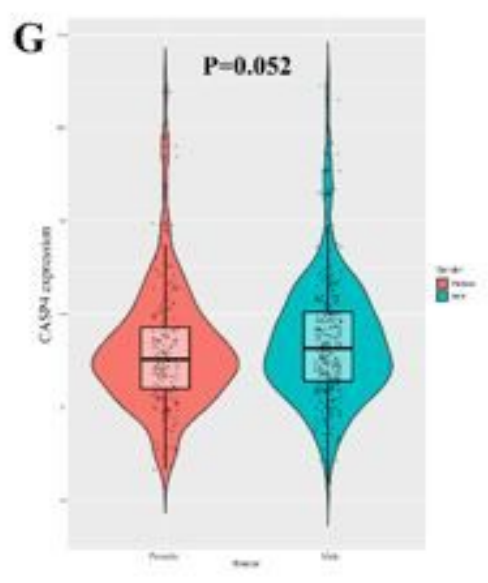

\section{Figure 4}

Clinicopathological significance of CASP4 expression. Expression of CASP4 in different (A) pathological grades, (B) clinical stages, (C) T stages, (D) N stages, (E) M stages, (F) age, and (G) sex. 

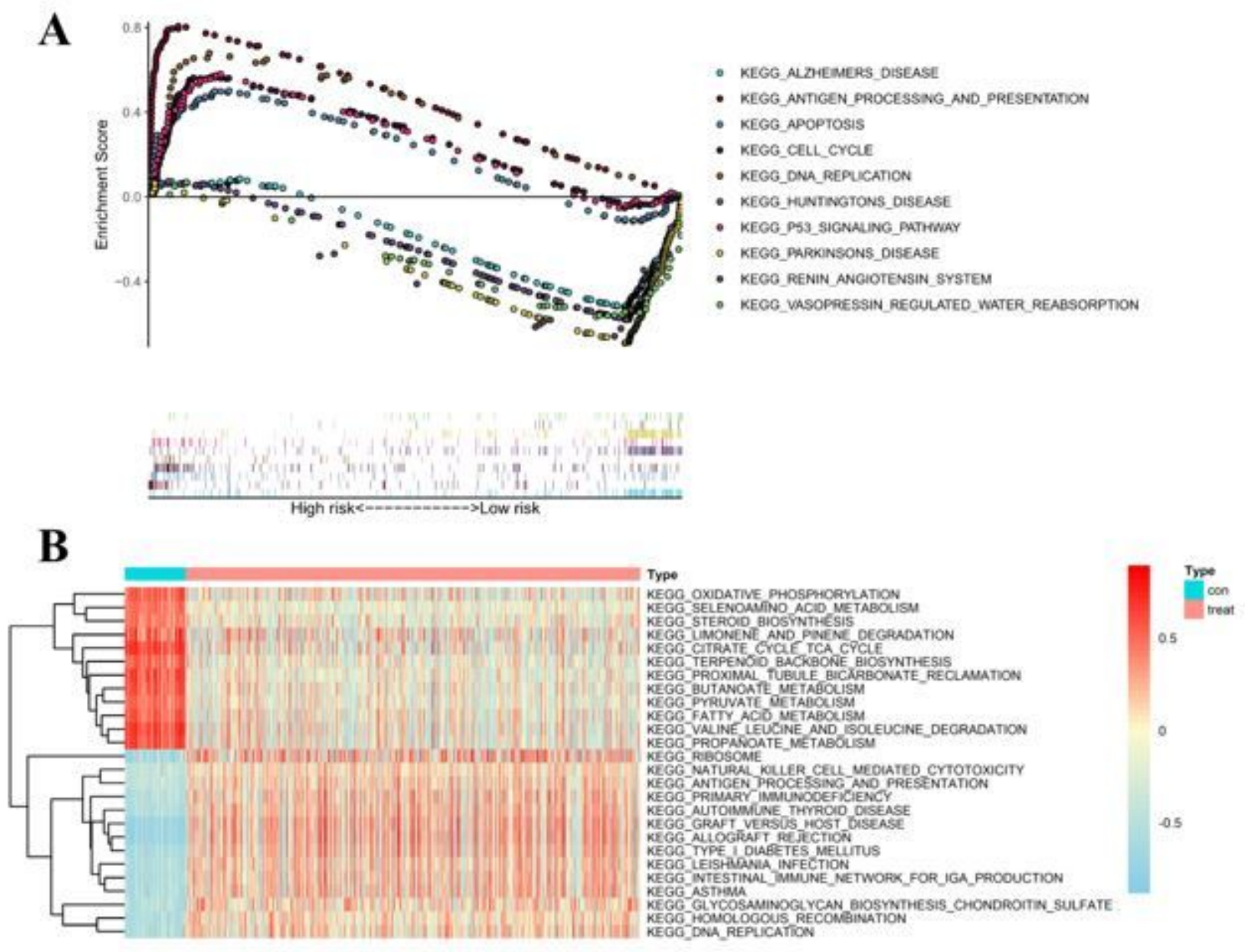

Figure 5

Gene set enrichment analysis (GSEA, A) and gene set variation analysis (GSVA, B) of CASP4 in the TCGA dataset. Only signaling pathways with $\log$ (foldchange) $>0.2$ are shown. 


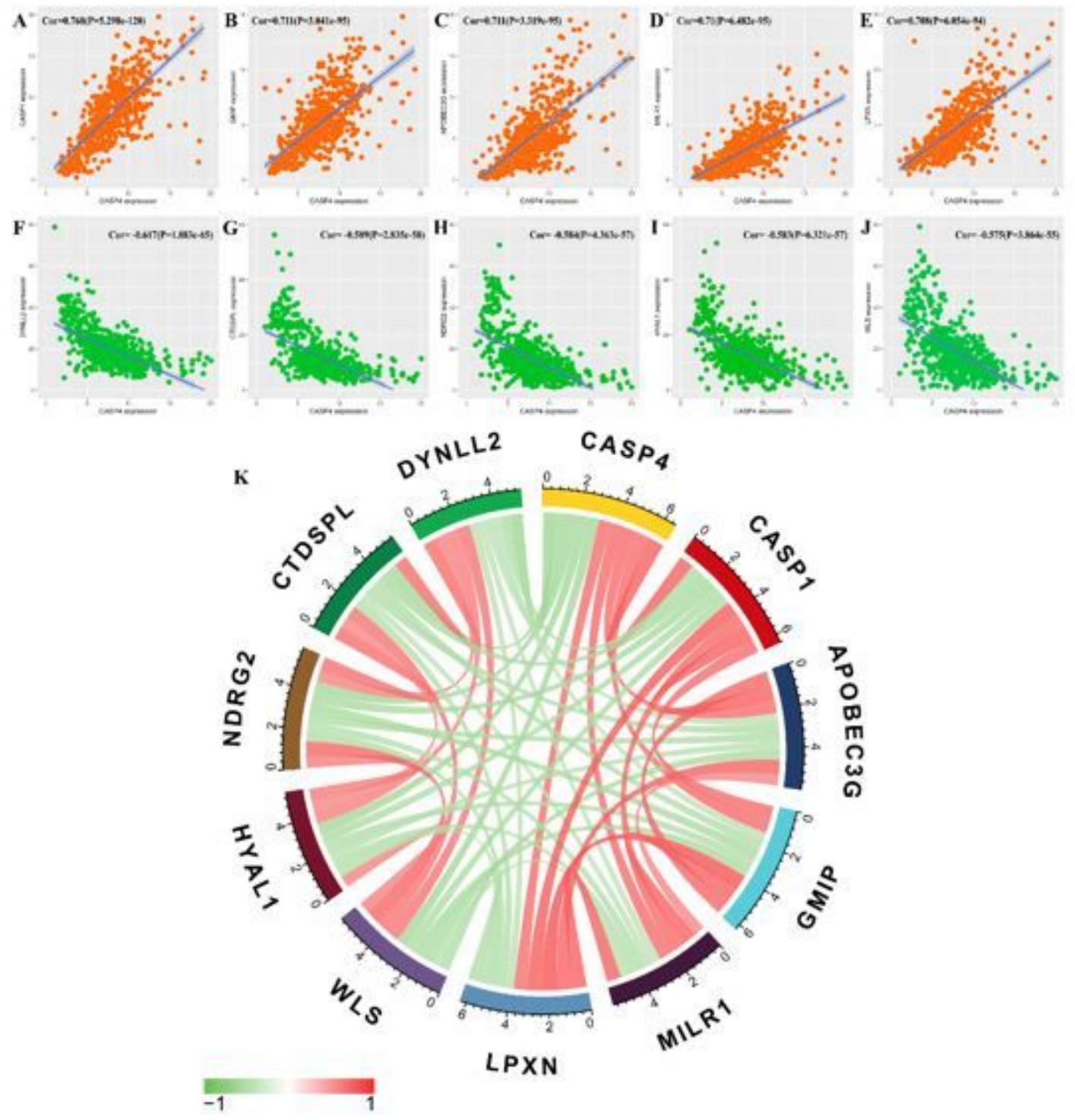

Figure 6

Relationship between CASP4 and the expression of other genes. (A) CASP1; (B) GMIP; (C) APOBEC3G; (D) MILR1; (E) LPXN; (F) DYNLL2; (G) CTDSPL; (H) NDRG2; (I) HYAL1; (J) WLS; (K) Circle diagram of the expression relationship between genes.

\section{Supplementary Files}

This is a list of supplementary files associated with this preprint. Click to download.

- Supp.Figure2.docx

- Supp.Figure1.docx 